\title{
Placenta-derived mesenchymal stem cells ameliorate lipopolysaccharide-induced inflammation in RAW264.7 cells and acute lung injury in rats
}

\author{
WEN YUAN $^{1 *}$, HENG-YA SONG $^{2 *}$, JIE XIONG $^{3}$, WAN-LI JIANG ${ }^{2}$, \\ GAN-JUN KANG ${ }^{2}$, JIE HUANG ${ }^{2}$ and SONG-PING XIE ${ }^{2}$
}

\author{
${ }^{1}$ Department of Laboratory Medicine, Wuhan Children's Hospital, Wuhan Maternal and Child Healthcare Hospital, \\ Tongji Medical College, Huazhong University of Science and Technology, Wuhan, Hubei 430016; \\ ${ }^{2}$ Department of Thoracic Surgery, Renmin Hospital of Wuhan University, Wuhan, Hubei 430060; \\ ${ }^{3}$ Department of Immunology, School of Basic Medical Science, Wuhan University, Wuhan, Hubei 430071, P.R. China
}

Received September 9, 2019; Accepted April 14, 2020

DOI: $10.3892 / \mathrm{mmr} .2020 .11231$

\begin{abstract}
Acute lung injury (ALI) is a severe lung syndrome with high morbidity and mortality, due to its complex mechanism and lack of effective therapy. The use of placenta-derived mesenchymal stem cells (pMSCs) has provided novel insight into treatment options of ALI. The effects of pMSCs on lipopolysaccharide (LPS)-induced inflammation were studied using a co-culture protocol with LPS-stimulated RAW264.7 cells. An LPS-induced ALI Sprague-Dawley rat model was developed by intravenously injecting $7.5 \mathrm{mg} / \mathrm{kg} \mathrm{LPS}$, and intratracheal instillation of $1 \times 10^{5} \mathrm{pMSCs}$ was performed after administration of LPS to investigate the therapeutic potential of these cells. pMSCs ameliorated LPS-induced ALI, as suggested by downregulated pro-inflammatory cytokine tumor necrosis factor- $\alpha$ and increased anti-inflammatory cytokine interleukin-10 in both cell and animal models. Moreover, the protein and leukocyte cells in bronchoalveolar lavage fluid decreased at a rapid rate after treatment with pMSCs. Histopathology demonstrated that pMSCs alleviated the infiltration of inflammatory cells, pulmonary hyperemia and hemorrhage, and interstitial edema. In addition, pMSC reduced the LPS-induced expression of C-X-C motif chemokine ligand 12 in RAW264.7 macrophages and in lung tissue
\end{abstract}

Correspondence to: Dr Song-Ping Xie or Dr Jie Huang, Department of Thoracic Surgery, Renmin Hospital of Wuhan University, 238 Jiefang Road, Wuchang, Wuhan, Hubei 430060, P.R. China

E-mail: songping0428@126.com

E-mail: doctor_huangjie@126.com

*Contributed equally

Key words: acute lung injury, acute respiratory distress syndrome, stem cell therapy, placenta-derived mesenchymal stem cells, rat of ALI rats. This demonstrated that pMSCs are therapeutically effective in LPS-induced ALI.

\section{Introduction}

Both acute lung injury (ALI) and acute respiratory distress syndrome (ARDS) are severe clinical conditions accompanied by interstitial edema and infiltration of inflammatory cells, which result in progressive acute respiratory failure (1-3). A previous systematic review reported that the mortality rate of pediatric ARDS (PARDS) was $24 \%$, with one-quarter of patients developing new morbidities after PARDS due to residual organ dysfunction and complications related to treatments $(4,5)$. Radiation-induced lung injury commonly occurs in patients receiving radiotherapy for thoracic cancer (6). Inhaled corticosteroids and other anti-inflammatory drugs are effective in patients with inflammatory lung disorders. However, their long-term use is associated with severe side effects (7). While ARDS mortality has moderately declined with improved ventilator management and fluid management, it has remained as high as 20 and $40 \%$ in clinical studies, respectively (2).

The therapeutic potential of mesenchymal stem cells (MSCs) for ARDS has been evaluated in previous clinical trials $(8,10)$. It was demonstrated that a single intravenous infusion of allogeneic bone marrow (BM)-MSCs was well tolerated in nine patients with moderate to severe ARDS in a previous clinical trial (NCT01775774) (8). However, the clinical use of BM-MSCs was hindered by low cell numbers on harvest (9). Another previous clinical study (NCT01902082) reported that intravenous administration of allogeneic adipose-derived MSCs was safe and feasible but inefficient in the treatment of ARDS (10). Compared with BM-MSCs, placenta-derived MSCs (pMSCs) are more easily obtained in large numbers $(11,12)$. As pMSCs have similar properties and functions as BM-MSCs, these cells have become a promising alternative source of MSCs in research and clinical applications (12). A previous study demonstrated that treatment with pMSCs was protective against the development of bronchiolitis obliterans in a murine 
model(13). Evidence has shown that the progress of pulmonary inflammation is closely related to the phenotype and function of macrophages $(14,15)$. pMSCs can regulate macrophage differentiation from the pro-inflammatory type to the anti-inflammatory type (16). However, to the best of the authors' knowledge, the therapeutic potential of pMSC delivery in ALI treatment has not been studied to date.

C-X-C motif chemokine 12 (CXCL12) belongs to the family of CXC chemokines. Diallyl trisulfide, a garlic-derived organosulfur compound, markedly reduced the expression of CXCL12 in lipopolysaccharide (LPS)-stimulated BV2 microglial cells, demonstrating its anti-inflammatory effects against LPS stimulation (17). The administration of CXCL12-neutralizing antibodies delayed disease onset or prevented disease progression in cancer, inflammatory bowel diseases and ALI (18). Thus, CXCL12 may act as an inflammatory cytokine in LPS-induced ALI.

ALI and ARDS often develop as a complication of severe sepsis, particularly after infection with Gram-negative bacteria (19). LPS-induced endotoxemia triggers the secretion of pro-inflammatory cytokines and is extensively used to establish an ALI animal model (20). In the present study, pMSCs were used to treat LPS-induced RAW264.7 macrophages and the effects of intratracheal delivery of pMSCs on LPS-induced ALI in Sprague-Dawley rats were investigated.

\section{Materials and methods}

Isolation and culture of pMSCs. pMSCs were isolated from chorionic villi in placenta of Sprague-Dawley rats, which were anesthetized by intraperitoneal anesthesia with $2 \%$ pentobarbital sodium (30 mg/kg). Rats were sacrificed using a supraphysiological dose of pentobarbital sodium $(100 \mathrm{mg} / \mathrm{kg})$. Briefly, placental tissue was dissected, then thoroughly washed with ice-cold sterile PBS (Gibco; Thermo Fisher Scientific, Inc.) containing antibiotics. After removing the amniotic membrane, the underlying chorionic villi were minced into a paste-like consistency. Then, $10 \mathrm{~g}$ tissue samples were enzymatically digested with trypsin and neutral protease for $60 \mathrm{~min}$ at $37^{\circ} \mathrm{C}$. Digestion was terminated with the addition of DMEM containing 10\% FBS (both from Gibco; Thermo Fisher Scientific, Inc.). The suspension was filtered using a 200 mesh sieve, collected and centrifuged at $1,000 \times \mathrm{g}$ for $10 \mathrm{~min}$ at $4^{\circ} \mathrm{C}$. The supernatant was discarded, and the pellet was resuspended in DMEM containing $10 \%$ FBS and plated in T25 Flasks (Corning, Inc.) in a $5 \% \mathrm{CO}_{2}$ air incubator at $37^{\circ} \mathrm{C}$ (CB 170; BINDER GmbH). pMSCs were identified by flow cytometry (CytoFLEX; Beckman Coulter, Inc.) and the detection of the following cell surface markers: CD11b/c (OX42, PE; cat. no. 12-0110-80; eBioscience; Thermo Fisher Scientific, Inc.); CD44 (OX-49, FITC; cat. no. MA5-17522; Thermo Fisher Scientific, Inc.); CD45 (OX1, APC; cat. no. 47-0461-80; eBioscience; Thermo Fisher Scientific, Inc.); CD90 (HIS51, FITC; cat. no. 03013-50; BioGems; PeproTech, Inc.); CD29 (HMb1-1, FITC; cat. no. 11-0291-80; eBioscience; Thermo Fisher Scientific, Inc.); CD31 (TLD-3A12, FITC; cat. no. MA5-16952; Thermo Fisher Scientific, Inc.). The unstained control was used as a negative control in this study. After incubation in the dark at $4^{\circ} \mathrm{C}$ for $30 \mathrm{~min}$, CytExpert software (version 2.0; Beckman Coulter, Inc.) was used for analysis.
Immunofluorescence. The cell density was adjusted to $3 \times 10^{6} / \mathrm{ml}$ and the cells were laid in the glass bottom dishes specially designed for laser confocal microscopy. Cells were fixed with $4 \%$ paraformaldehyde for $20 \mathrm{~min}$ at $4^{\circ} \mathrm{C}$ and permeabilized with $0.1 \%$ Triton X-100 for 10 min, blocked with $3 \%$ BSA, and sealed for $60 \mathrm{~min}$ at room temperature. After incubation overnight with anti-CD44 antibody (1:200; cat. no. ab189524; Abcam), the cells were rinsed thoroughly and treated with anti-rabbit antibodies (1:200; cat. no. BA1032; Boster Biological Technology), respectively. Nuclei were stained using $0.3 \mu \mathrm{M}$ DAPI (cat. no. C1002; Beyotime Institute of Biotechnology).

Osteogenic and adipogenic differentiation of pMSCs. The third generation of pMSCs were seeded at $1 \times 10^{5}$ cells $/ \mathrm{cm}^{2}$ on a 24-well plate (Merck KGaA). When the density of cells reached $80 \%$, the complete medium was removed and cells were induced with an osteogenic induction medium (DMEM with 10\% FBS; $0.1 \mu \mathrm{M}$ dexamethasone; $10 \mathrm{mM} \beta$-glycerophosphate sodium; $50 \mu \mathrm{M}$ ascorbic acid). Cells were cultured for 3 weeks and the medium was changed every $72 \mathrm{~h}$. After 3 weeks, differentiated pMSCs were stained with aqueous $0.5 \%(\mathrm{v} / \mathrm{v})$ Alizarin Red S (Beijing Solarbio Science \& Technology Co, Ltd.) for $30 \mathrm{~min}$ at room temperature. For adipogenic induction, the cells were plated in DMEM supplemented with $10 \%$ FBS, $200 \mu \mathrm{M}$ indomethacin, $1 \mu \mathrm{M}$ dexamethasone, $0.5 \mathrm{~mm}$ isobutyl methylxanthine and $0.5 \mu \mathrm{g} / \mathrm{ml}$ insulin. After 10 days, the differentiation was verified using Oil Red O staining for $10 \mathrm{~min}$ at room temperature (Sigma-Aldrich; Merck KGaA).

Cell culture and stimulation. RAW264.7 cells (cat.no. CL-0190; Procell Life Science \& Technology Co., Ltd.) were cultured in DMEM supplemented with $10 \% \mathrm{FBS}, 100 \mathrm{U} / \mathrm{ml}$ penicillin and $100 \mu \mathrm{g} / \mathrm{ml}$ streptomycin. The cell cultures were maintained in a $5 \% \mathrm{CO}_{2}$ air incubator at $37^{\circ} \mathrm{C}$. RAW264.7 cells were then seeded and cultured in six-well plates at a density of $5 \times 10^{5}$ cells/well, followed by treatment with LPS at different concentrations (1, 2 or $5 \mu \mathrm{g} / \mathrm{ml})$ for different time periods $(0,0.5,1,2,4$ or $24 \mathrm{~h})$, and tumor necrosis factor- $\alpha$ (TNF- $\alpha$ ) levels were detected by ELISA (cat. no. E-EL-M0049c; Elabscience Biotechnology, lnc.) to determine if the LPS-induced RAW264.7 macrophage inflammatory model was established. To detect the therapeutic effects of pMSCs in the RAW264.7 macrophage inflammation model in vitro, RAW264.7 cells and pMSCs were co-cultured at $37^{\circ} \mathrm{C}$ for $48 \mathrm{~h}$ in two chambers using Millicell (Corning Inc.) to prevent contact between the cells.

TNF- $\alpha$ and interleukin (IL)-10 ELISA. Cell-free supernatants were collected, and IL-10 and TNF- $\alpha$ levels were measured using IL-10 (cat. no. SEA056Ra, Wuhan USCN Business Co., Ltd.) and TNF- $\alpha$ (cat. no. E-EL-R0019c; Elabscience Biotechnology, Inc.) ELISA kits according to the manufacturers' instructions.

Reverse transcription-quantitative PCR (RT-qPCR). Total RNA was isolated from RAW264.7 cells using TRIpure (cat. no. RN0101; Aidlab Biotechnologies Co., Ltd.) and reverse transcribed into cDNA using the HiScript Reverse Transcriptase (RNase H) (cat. no. R101-01/02; Vazyme Biotech Co., Ltd.) at the following conditions: $25^{\circ} \mathrm{C} 5 \mathrm{~min} ; 50^{\circ} \mathrm{C}$ $15 \mathrm{~min} ; 85^{\circ} \mathrm{C} 5 \mathrm{~min}$. PCR amplification was independently 
performed with a SYBR Green PCR kit (cat. no. Q111-02; Vazyme Biotech Co., Ltd.) using the Applied Biosystems 7500 real-time PCR system (Applied Biosystems QuantStudio 6; Applied Biosystems; Thermo Fisher Scientific, Inc.) in triplicate. The thermocycling conditions of the PCR were as follows: $5 \mathrm{~min}$ at $95^{\circ} \mathrm{C}, 15 \mathrm{sec}$ at $95^{\circ} \mathrm{C}$ and $1 \mathrm{~min}$ at $60^{\circ} \mathrm{C}$ for 40 cycles, then $5 \mathrm{~min}$ at $72^{\circ} \mathrm{C}$. The reaction specificity was controlled by post-amplification melting curve analysis and gel electrophoresis. The expression fold changes were analyzed using the $2^{-\Delta \Delta C q}$ relative quantification method (21). Primer sequences were as follows: IL-10-forward, 5'-GCTGGACAACATACT GCTAACCG-3'; IL-10-reverse, 5'-CACAGGGGAGAAATC GATGACAG-3'; TNF- $\alpha$-forward, 5'-CGTCAGCCGATTTGC TATCT-3'; TNF- $\alpha$-reverse, 5 '-CGGACTCCGCAAAGTCTA AG-3'; CXCL12-forward, 5'-TCAACACTCCAAACTGTG CCCTTCA-3'; CXCL12-reverse, 5'-GCCTTTCTCTTCTTC TGTCGCTTCT-3'; $\beta$-actin-forward, 5'-CACGATGGAGGG GCCGGACTCATC-3' and $\beta$-actin-reverse, 5'-TAAAGACCT CTATGCCAACACAGT - 3 '.

Western blotting. Cells were lysed in RIPA lysis buffer (Beyotime Institute of Biotechnology) on ice. Protein concentrations were determined using the BCA kit (Thermo Fisher Scientific, Inc.). The sample proteins ( $40 \mu \mathrm{g} / \mathrm{lane})$ were separated via SDS-PAGE on 15\% gels and transferred to a PVDF membrane (EMD Millipore). The membranes were then blocked with TBS containing 5\% non-fat dried milk and $0.1 \%$ Tween-20 at $4^{\circ} \mathrm{C}$ for $2 \mathrm{~h}$. After blocking, the membranes were incubated with a primary anti-CXCL12 antibody $(1: 1,000$; Abcam; cat. no. ab9797) overnight at $4^{\circ} \mathrm{C}$ with gentle shaking and subsequently incubated with HRP-conjugated anti-rabbit IgG secondary antibody (1:50,000; Wuhan Boster Biological Technology, Ltd.; cat. no. BA1054) for $2 \mathrm{~h}$ at room temperature. The bands were detected using enhanced chemiluminescence western blotting detection reagents (Thermo Fisher Scientific, Inc.). GAPDH (1:1,000; cat. no. AB-P-R 001; Goodhere Biological Technology) was used as a loading control, and the band density was measured using ImageJ (version 1.52a; National Institutes of Health).

Rat model of ALI. All animal procedures were approved by The Ethics Committee of Clinical Research, Renmin Hospital of Wuhan University (reference no. WDRY2018-K048). All animal experiments were performed in compliance with the Guidelines for Proper Conduct of Animal Experiments established by the Science Council. A total of 15 adult male Sprague-Dawley rats (aged 6-8 weeks; weight, 280-350 g) were provided by Hunan SJA Laboratory Animal Co., Ltd. The rats were housed in a specific pathogen-free environment at $18-26^{\circ} \mathrm{C}$ in the dark, with $40-70 \%$ humidity, and free access to food and water. These rats were randomly divided into three groups: A normal control (NC) group injected with $0.5 \mathrm{ml}$ PBS; an LPS-induced ALI group intravenously receiving $7.5 \mathrm{mg} / \mathrm{kg}$ LPS dissolved in $0.5 \mathrm{ml}$ sterile saline solution (22); and in the LPS + pMSCs group, rats were anesthetized using $2 \%$ pentobarbital $(30 \mathrm{mg} / \mathrm{kg})$, and $1 \times 10^{5} \mathrm{pMSCs}$ intratracheal instillation was performed after administration of LPS for $1 \mathrm{~h}$ (pMSCs group). After 3 days, the lung tissue and the bronchoalveolar lavage fluid (BALF) samples were collected $(23,24)$. There were five rats in each group.
Analysis of BALF. BALF was collected and centrifuged at $600 \mathrm{x} \mathrm{g}$ for $5 \mathrm{~min}$ at $25^{\circ} \mathrm{C}$, for quantification of total protein content in the BALF supernatant standard BALF collection was performed, as described previously (25). The protein concentration was measured using a BCA Protein Assay kit (Beyotime Institute of Biotechnology) following the manufacturer's instructions. BALF cytokine levels were examined using IL-10 (cat. no. SEA056Ra, Wuhan USCN Business Co., Ltd.) and TNF- $\alpha$ (cat. no. E-EL-R0019c, Elabscience Biotechnology, Inc.) ELISA kits.

Cell counts and Giemsa staining. The number of white blood cells in BALF was counted using a Bowman plate. The alveolar lavage fluid was coated with glass slides and stained with Giemsa (reagent 1 solution for $1 \mathrm{~min}$ and reagent 2 for 6-8 mins at room temperature), then the stained cells were observed with a light microscope at magnification, $\mathrm{x} 100$ and $\times 200$.

Histopathological observation of lungs. The lower lobe of the right lung was collected from the rats, fixed in $10 \%$ formaldehyde solution for $48 \mathrm{~h}$ at room temperature, embedded in paraffin, dehydrated, cut into 5- $\mu \mathrm{m}$ sections and observed under an optical microscope (magnification, x100 and x200) after hematoxylin-eosin (HE) staining for $5 \mathrm{~min}$ at room temperature.

Immunohistochemical analysis. Paraffin-embedded tissues were sectioned at $5 \mu \mathrm{m}$, mounted onto glass slides and dewaxed, then rehydrated in a descending alcohol gradient $(100,95,90,80,70$ and $50 \%)$. Antigen retrieval was performed by microwaving at high power in $10 \mathrm{mM}$ sodium citrate buffer, pH 6.0 for $20 \mathrm{~min}$. To block the non-specific binding of antibodies, each slide was blocked with $10 \%$ normal goat serum (cat. no. AR1009; Boster Biological Technology) for $30 \mathrm{~min}$ at room temperature. Immunostaining was performed by incubation with an anti-CXCL12 antibody (1:200, cat. no. ab9797; Abcam) at $4^{\circ} \mathrm{C}$ overnight. Slides were then washed in PBS and incubated with secondary antibody (anti-rabbit detection system; 1:200; cat. no. GB23303; Wuhan Servicebio Technology Co., Ltd.) for $30 \mathrm{~min}$ at $37^{\circ} \mathrm{C}$. Tissues were stained with 3, 3-diaminobenzidine for $5 \mathrm{~min}$ at room temperature and counterstained with hematoxylin for $1 \mathrm{~min}$ at room temperature. Photomicrographs were taken using an Olympus BX53 microscope (Olympus Corporation), at magnification, $\mathrm{x} 100$ and $\mathrm{x} 400$. Image Pro Plus version 6.0 (Media Cybernetics, Inc.) was used to determine staining intensity.

Statistical analysis. All data were analyzed using GraphPad Prism version 5.0 software (GraphPad Software, Inc.) and presented as the mean $\pm \mathrm{SD}$ of three independent experiments. One-way ANOVA followed by Newman-Keuls Multiple Comparison post hoc test were used when appropriate. $\mathrm{P}<0.05$ was considered to indicate a statistically significant difference.

\section{Results}

Isolation and identification of pMSCs. pMSCs from rat embryos were isolated and cultured, which were passed to the P2 generation and adherent in a serum-containing medium and showed a spindle-like shape (Fig. 1A). Phenotypic analysis 
A

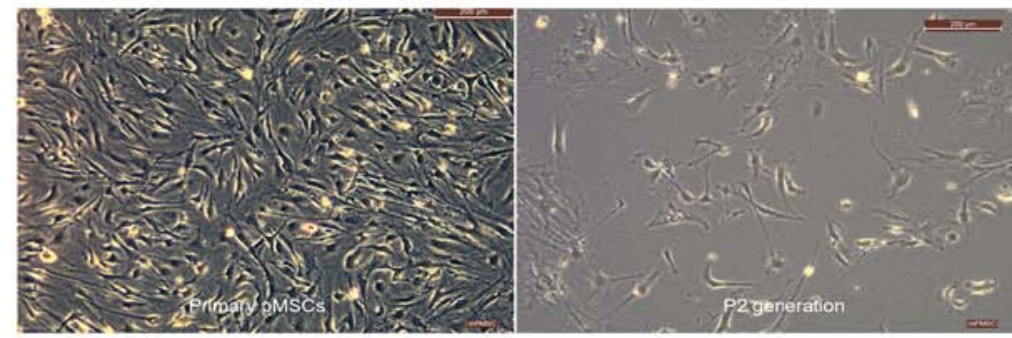

B
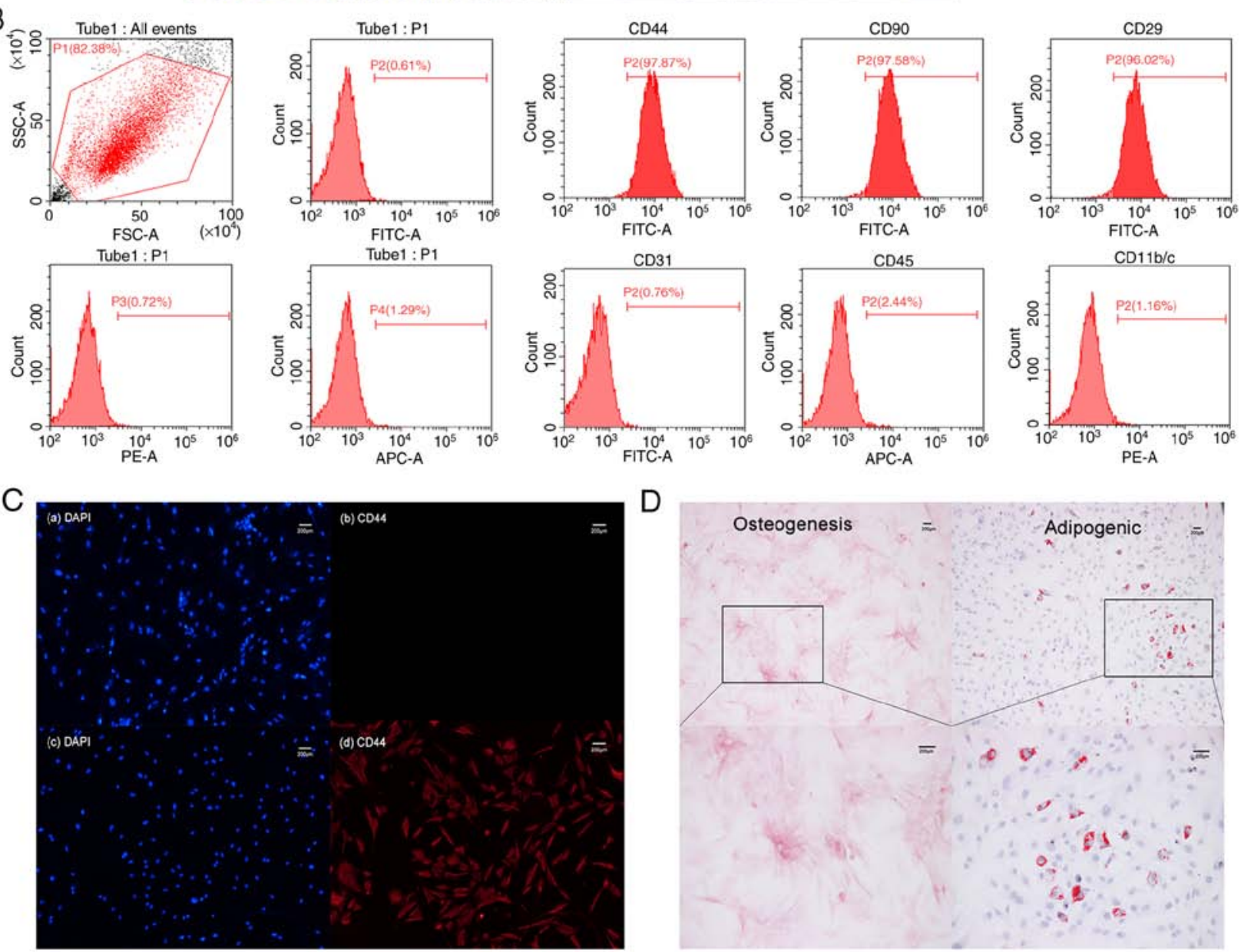

Figure 1. Isolation and identification of rat pMSCs. (A) Primary culture and P2 generation cell morphology (magnification, x100). (B) The three on the left panels are unstained negative controls of flow cytometry analysis, while the six on the right are the quantitative analysis of CD44, CD90, CD29, CD31, CD45 and CD11b/c. (C) Immunofluorescence staining of pMSCs. (a and b) Control group. (c and d) Experimental group. Non-specific nuclear staining is shown in blue on the left-hand side, and specific CD44 staining is in red on the right-hand side. (D) Multi-lineage differentiation capacity of pMSCs. Osteogenesis differentiation was demonstrated by Alizarin Red S staining (magnification, x100 and x200), while adipogenic differentiation was demonstrated by Oil Red O staining (magnification, x100 and x200). pMSC, placenta-derived mesenchymal stem cell; PE, phycoerythrin; APC, allophycocyanin.

was carried out by flow cytometry. CD44, CD90 and CD29 were $97.87,97.58$ and $96.02 \%$, respectively, while CD31, CD45, CD11b/c were negative (Fig. 1B). This was consistent with the expected pMSC phenotype $\mathrm{CD} 44^{+} \mathrm{CD} 90^{+} \mathrm{CD} 29^{+} \mathrm{CD} 3$ $1^{-\mathrm{CD}} 45^{-\mathrm{CD}} 1 \mathrm{~b} / \mathrm{c}^{-}$. Subsequently, immunofluorescence staining was used to evaluate specific expression of the CD44 marker in pMSCs (Fig. 1C). The primary antibody was replaced with PBS in the control group, while the CD44 antibody of anti-mouse was replaced in the experimental group. Although the staining result was similar in non-specific nuclear staining of both groups, specific CD44 staining in the experimental group was much stronger than that in the control group. Thus, cells isolated from rat embryos were indeed pMSCs that expressed CD44. The potential pluripotent ability of pMSCs was confirmed by osteogenic and adipogenic differentiation in vitro (Fig. 1D), this indicated that $\mathrm{pMSCs}$ have multilineage differentiation ability in the cultured condition.

Therapeutic effects of pMSCs in RAW264.7 macrophage inflammation model in vitro. Before establishing the inflammatory model, the optimally induced concentration of LPS was evaluated using ELISA and to establish an LPS-induced RAW264.7 macrophage inflammation model. When the concentration of the pro-inflammatory cytokine TNF- $\alpha$ reached the maximum, the optimum condition was achieved. As presented in Fig. 2A, the optimal concentration was $5 \mu \mathrm{g} / \mathrm{ml}$ with co-culturing for $4 \mathrm{~h}$. The inflammation model of RAW264.7 macrophages by this optimal condition for $4 \mathrm{~h}$ was 
A
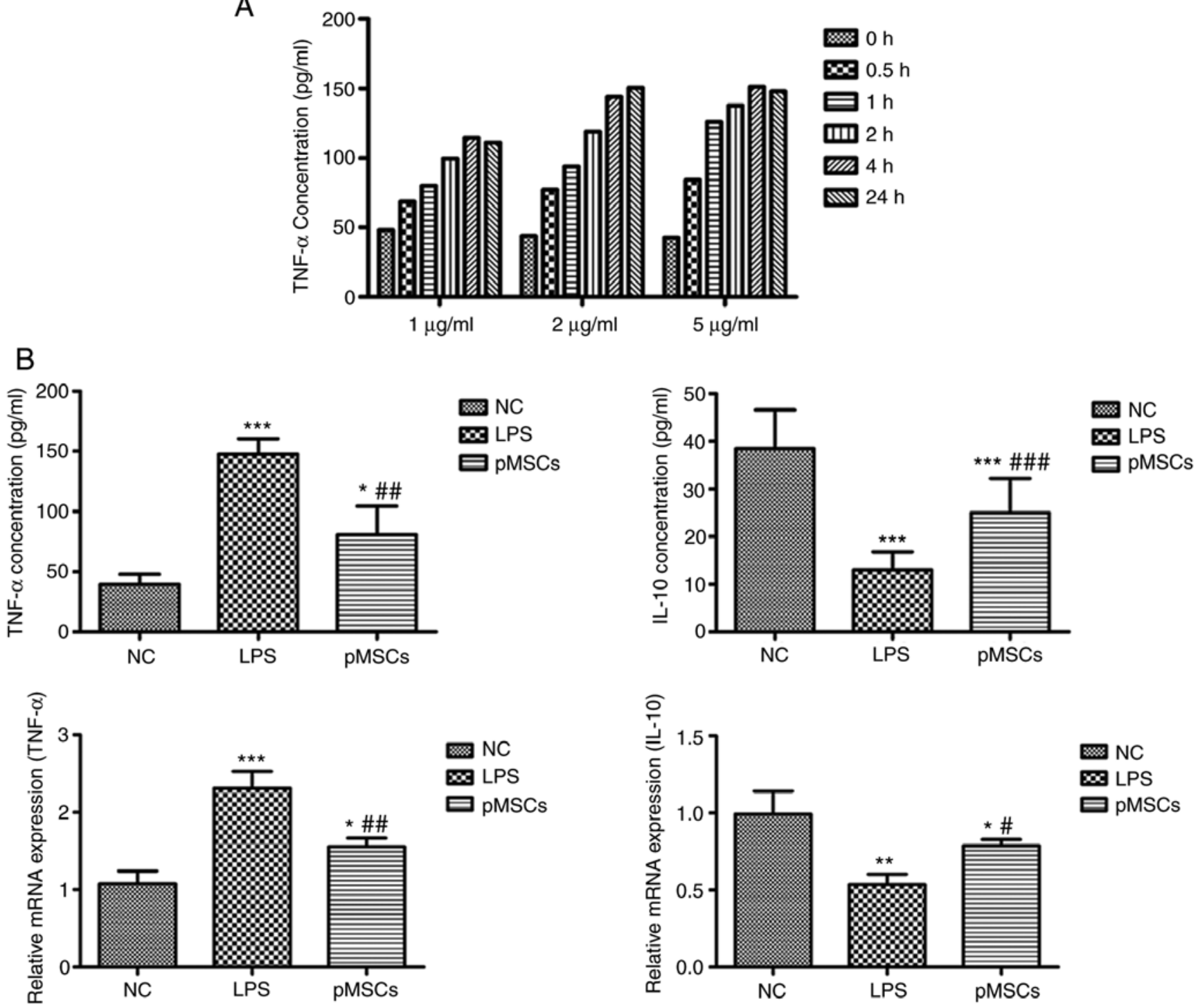

Figure 2. Expression changes of inflammatory cytokines in vitro. (A) Changes in the concentration of TNF- $\alpha$ in the presence of LPS. (B) Co-cultures with pMSCs for $48 \mathrm{~h}$ during LPS-induced acute lung injury. TNF- $\alpha$ and IL-10 were measured by ELISA and reverse transcription-quantitative PCR in vitro. The absorbance was measured at $450 \mathrm{~nm}$ using a microplate reader. The NC group denotes RAW264.7 cells (1x105); the LPS group, cells treated with LPS; and the pMSC group, cells treated with LPS + pMSCs $\left(1 \times 10^{5}\right)$. Data are presented as the mean $\pm \mathrm{SD} .{ }^{*} \mathrm{P}<0.05,{ }^{* * *} \mathrm{P}<0.01$ and ${ }^{* * * *} \mathrm{P}<0.001$ vs. $\mathrm{NC} ;{ }^{\#} \mathrm{P}<0.05,{ }^{\# \#} \mathrm{P}<0.01$ and ${ }^{\# \#} \mathrm{P}<0.001$ vs. LPS. $\mathrm{n}=3$ in each group. pMSC, placenta-derived mesenchymal stem cell; TNF- $\alpha$, tumor necrosis factor- $\alpha$; LPS, lipopolysaccharide; NC, normal control; IL, interleukin.

then induced. A total of three groups were formed: RAW264.7 cells as the control (NC group), RAW264.7 cells treated with LPS (LPS group), and RAW264.7 cells treated with LPS + pMSCs (pMSCs group). The expressions of cytokines TNF- $\alpha$ and IL-10 were detected. As presented in Fig. 2B, pMSCs reduced the expression of TNF- $\alpha$ and increased IL-10 in the cell inflammatory model. These results provide evidence for the direct involvement of the therapeutic effect of pMSCs in LPS-induced injury.

Influence of pMSCs on lung histopathology. An LPS-induced ALI animal model was established to determine whether pMSCs had a protective effect against LPS-induced ALI in vivo. The histopathology of rat lung tissue from each group was examined using HE staining (Fig. 3A). The HE staining of lung sections before administration of LPS showed no obvious lesions in the NC group. However, after LPS was administered, typical histological features of ALI were observed in the LPS group, including diffuse alveolar injury, massive pleomorphic leukocytes in the stroma, obvious hyperemia and hemorrhage in alveolar, remarkable interstitial edema, and thickened alveolar septum. Although the treatment of pMSCs improved the lung histopathology by reducing neutrophil infiltration and alleviating interstitial edema, there was still challenge compared with that of the NC group.

Effect of pMSCs on BALF inflammatory cells and proteins. As the hallmark of ALI, indicators of vascular leakage in BALF were assessed, typically the total leukocyte cell number and total protein concentration, which are common indicators to evaluate the severity of alveolar-capillary membrane injury. The pMSCs injected into the LPS-induced ALI rats caused a decrease in the balance of inflammatory cells and the total cell count was significantly reduced compared with the LPS group 
A
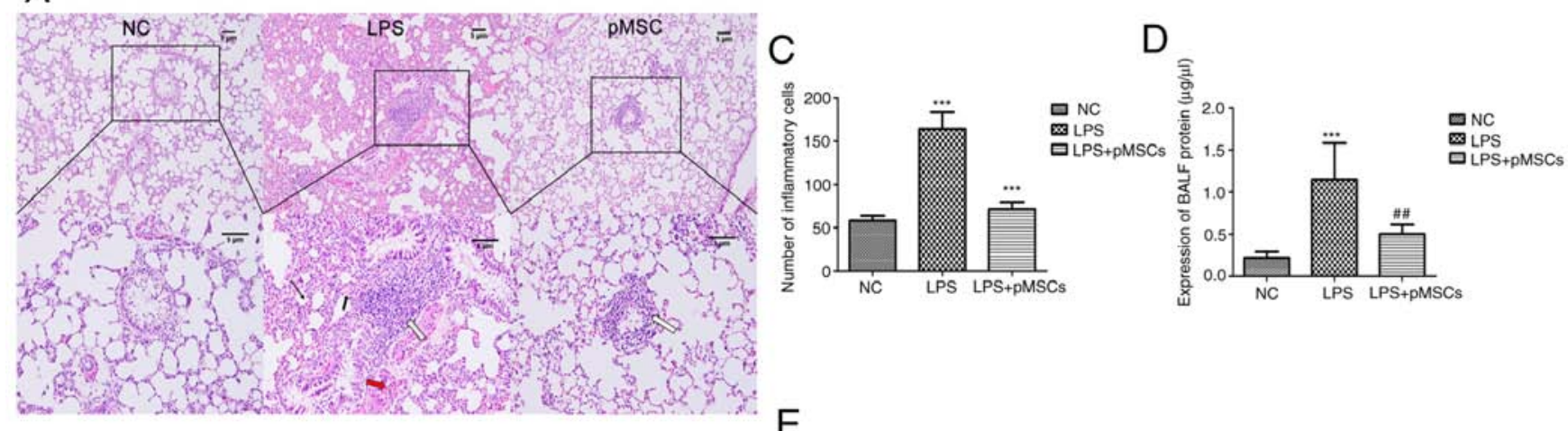

B
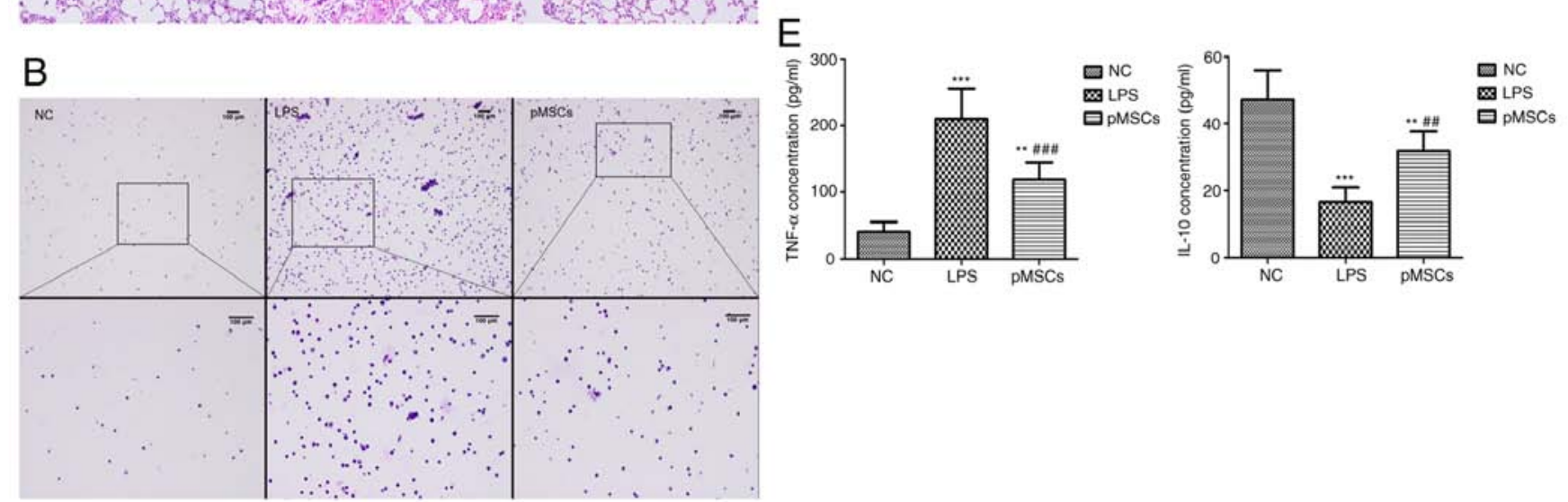

Figure 3. Therapeutic effects of intratracheal instillation of pMSCs in the acute lung injury model. (A) Representative hematoxylin-eosin staining of lung sections. A shrinking alveolar cavity (thin black arrow), inflammatory cells infiltrating in the interstitium of the lung (white arrow), pulmonary hemorrhage (red arrow) and alveolar septal thickening (bold black arrow) are presented. (B) Wright-Giemsa staining of inflammatory cells in BALF samples of the rats. (C) Enumeration of inflammatory cells in BALF samples. (D) Determination of BALF protein. (E) TNF- $\alpha$ and IL-10 in BALF were measured using ELISA kits. Data are presented as the mean $\pm \mathrm{SD}$. ${ }^{* *} \mathrm{P}<0.01$ and ${ }^{* * * *} \mathrm{P}<0.001$ vs. NC; ${ }^{\# \#} \mathrm{P}<0.01$ and ${ }^{\# \# t} \mathrm{P}<0.001$ vs. LPS. $\mathrm{n}=5$ in each group. NC, normal control; LPS, lipopolysaccharide; BALF, bronchoalveolar lavage fluid; pMSCs, placenta-derived mesenchymal stem cells; TNF- $\alpha$, tumor necrosis factor- $\alpha$; IL, interleukin.

(Fig. 3B and C). The protein in BALF was markedly increased in rats that received LPS compared with untreated rats $(\mathrm{P}<0.05$ vs. NC group). Moreover, a significant decrease in the protein of BALF was observed in the pMSCs group $(\mathrm{P}<0.05$ vs. LPS group; Fig. 3D).

Reduced TNF- $\alpha$ and increased IL-10 by pMSCs. TNF- $\alpha$ and IL-10 expression levels in BALF of the three groups were determined using ELISA. The average of the data was acquired after multiple measurements. The administration of pMSCs led to decreased TNF- $\alpha$ and increased IL-10 (Fig. 3E) during LPS-induced ALI, which suggested that pMSCs are an effective therapy for LPS-induced ALI.

pMSC downregulates CXCL12 in LPS-induced ALI. In our previous studies it was demonstrated that the activation of the CXCL12/CXCR4 axis played an important role in the metastasis and drug resistance in lung cancer (26-28). Moreover, CXCL12 could serve as pro-inflammatory chemokines as was reported in some previous studies $(17,18)$. To further investigate the mechanism underlying the effect of pMSCs on LPS-induced ALI, mRNA levels and protein expression of CXCL12 in LPS-induced RAW264.7 cells and LPS-induced ALI were measured. As presented in Fig. 4A and B, the LPS group showed a significantly higher expression of CXCL12 compared with the $\mathrm{NC}$ group $(\mathrm{P}<0.001)$. However, treatment with pMSCs reversed this effect, suggesting that pMSCs reduced the expression of CXCL12 in the RAW264.7 macrophage inflammatory model $(\mathrm{P}<0.05)$. To demonstrate the effects of pMSCs on histopathological changes in lung tissues in LPS-induced ALI rats, histological analysis was carried out using immunohistochemistry. The LPS group displayed very high expression of CXCL12, while the pMSCs group showed a marked reduction of CXCL12 (Fig. 4C and D). These data indicated that pMSC administration reduced the LPS-induced expression of CXCL12 in RAW264.7 macrophage and in lung tissue of ALI rats. Therefore, pMSCs significantly regulated levels of pro-inflammatory cytokines, such as TNF- $\alpha(\mathrm{P}<0.05)$ and anti-inflammatory IL-10 ( $\mathrm{P}<0.05)$, as well as accumulation of inflammatory cells and protein concentration in BALF. This suggested that pMSCs were effective in attenuating LPS-induced ALI.

\section{Discussion}

Despite the increased understanding of the pathogenesis of ALI and ARDS, the underlying mechanism remains to be investigated.Currently, there is evidence suggesting that macrophages are key factors in the pathogenesis of ALI/ARDS (29). Macrophages can be divided into pro-inflammatory M1-type and anti-inflammatory M2-type macrophages. While the M1-type can secrete TNF- $\alpha$ cytokines to promote the progress of inflammation, the M2-type can secrete IL-10 to inhibit the progress of inflammation (30). A previous study suggested that 

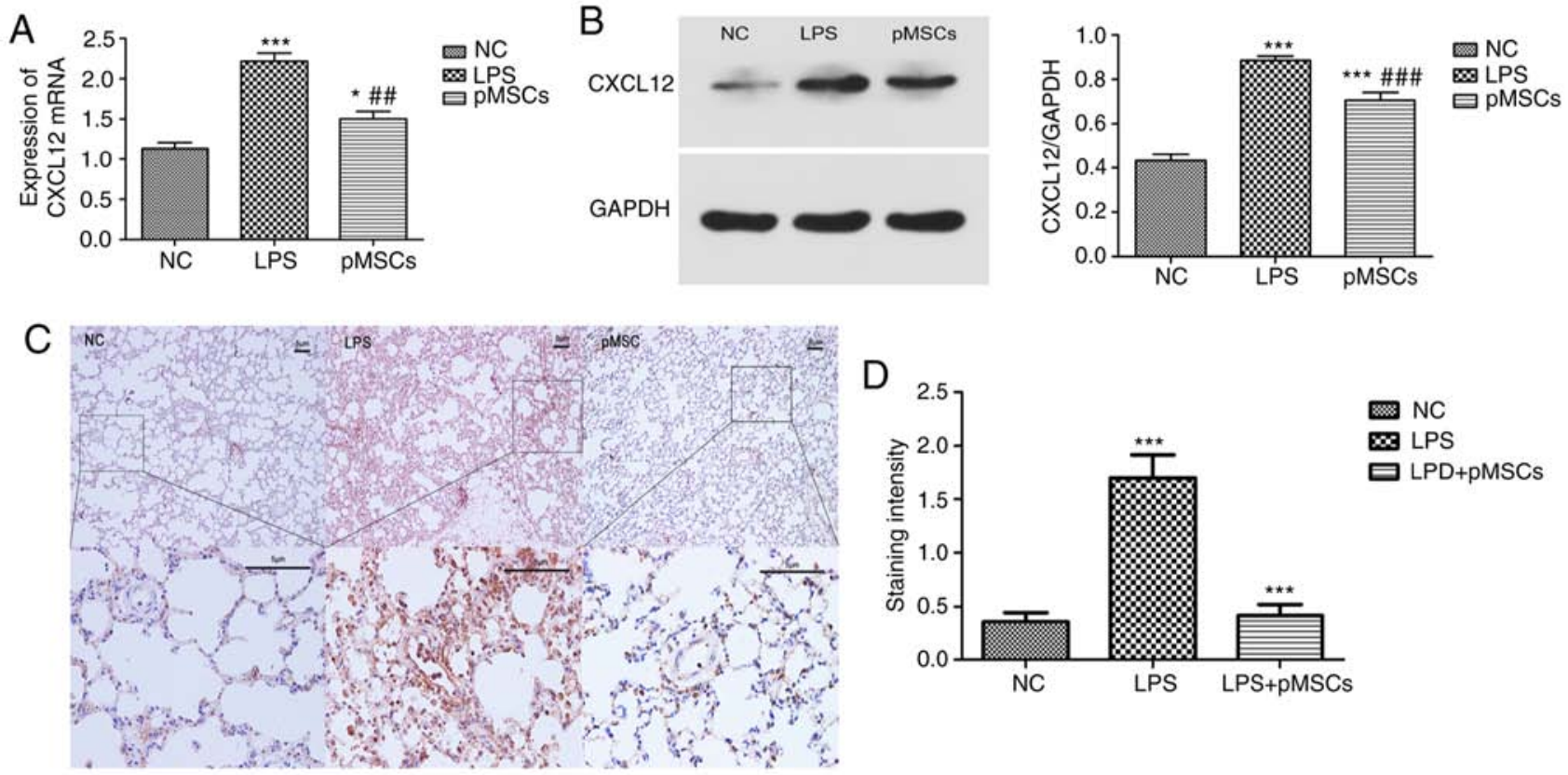

Figure 4. Expression of CXCL12 of LPS-induced RAW264.7 macrophages and lung tissue of acute lung injury rats following treatment with pMSCs. Expression of CXCL12 in RAW264.7 macrophages was measured by (A) RT-qPCR and (B) western blot analysis. $\beta$-actin and GAPDH were used as internal controls in RT-qPCR and western blotting, respectively. (C) Representative immunohistochemistry images of lung tissue from the three groups (magnifications, $\mathrm{x} 100$ and $\mathrm{x} 400$ ). (D) Staining intensity of CXCL12 of lung tissues from the three groups. Data are presented as the mean $\pm \mathrm{SD}$. ${ }^{*} \mathrm{P}<0.05$ and ${ }^{* * * *} \mathrm{P}<0.001 \mathrm{vs}$. NC; ${ }^{* \#} \mathrm{P}<0.01$ and ${ }^{\# \# \#} \mathrm{P}<0.001$ vs. LPS. $n=5$ in each group. RT-qPCR, reverse transcription-quantitative PCR; NC, normal control; LPS, lipopolysaccharide; CXCL12, C-X-C motif chemokine ligand 12; pMSCs, placenta-derived mesenchymal stem cells.

regulating the function of macrophages might be a promising therapeutic strategy against ALI/ARDS (14). The present study demonstrated that pMSCs could inhibit the inflammatory response by decreasing the secretion of TNF- $\alpha$ cytokines and increasing the IL-10 secretion in an LPS-induced RAW264.7 macrophage inflammatory model.

In the present study, pMSCs could inhibit the inflammatory response of LPS-induced RAW264.7 macrophages. Thus, the focus of the present study was to evaluate whether pMSCs had an effect on ALI in rats and how this therapeutic effect could be exerted. An ALI rat model was established using intravenous instillation of LPS. Pretreatment with pMSCs significantly reduced LPS-induced lung pathological changes, levels of pro-inflammatory cytokines and infiltration of pleomorphic leukocytes and total protein in BALF. Previous studies demonstrated that MSCs could be transplanted into rabbits or humans with beneficial effects and without immunological rejection $(31,32)$. In the present study, pMSC administration did not cause any adverse effect in rats and led to decreased TNF- $\alpha$ and increased IL-10 levels during LPS-induced ALI. A previous study demonstrated that IL-10 overexpression in umbilical cord MSCs enhanced their effects in E. coli pneumosepsis and increased macrophage function, which illustrated their therapeutic potential for infection-induced ARDS (33). Recently, it has been reported that erythropoietin produces protective effects against ALI in rats by increasing the levels of anti-inflammatory cytokine IL-10 (34). The present study demonstrated that pMSCs increased the IL-10 secretion of LPS-induced RAW264.7 macrophages. In addition, pMSC treatment increased IL-10 levels, which led to beneficial effects on LPS-induced ALI and enhanced the macrophage function. pMSCs reduced the
LPS-induced expression of CXCL12 in RAW264.7 macrophages and in lung tissue of ALI rats. Therefore, CXCL12 may act as an inflammatory cytokine in LPS-induced ALI and pMSCs could have a therapeutic effect on ALI through inhibition of CXCL12 expression.

In the present study, TNF- $\alpha$ expression was used to characterize the LPS-induced RAW264.7 macrophage inflammatory model. BALF inflammatory cells and protein, lung histopathology and pro-inflammatory cytokine TNF- $\alpha$ were used to confirm establishment of the LPS-induced ALI rat model. Although TNF- $\alpha$ is the most representative and commonly used inflammatory cytokine (35-37), other inflammatory cytokines, such as IL-1 $\beta$ (35), nuclear factor- $\kappa B$ (36) and IL-6 (37) should also be assessed to further characterize LPS-induced ALI. Several previous studies suggested that macrophages are a key component in the initiation and maintenance of the inflammatory response in ALI $(14,38,39)$. Thus, modulation of macrophage function might provide new therapeutic modalities for ALI. The LPS-induced RAW264.7 macrophage inflammatory model is a commonly used inflammatory model $(40,41)$. The results of the present study demonstrated that pMSCs could inhibit the inflammatory response of LPS-induced RAW264.7 macrophage inflammatory model in vitro. In accordance with other previous studies (42-44), the NC, LPS and LPS + pMSC groups were used both in vitro and in vivo. The effects of pMSC administration without LPS stimulation have not been evaluated in the present study.

In summary, the present study demonstrated that pMSCs reduced inflammation and protected against lung injury in the LPS-induced ALI rat model. However, the mechanisms and long-term effects need to be studied further. 


\section{Acknowledgements}

Not applicable.

\section{Funding}

The present study was supported by The National Natural Foundation of China (grant no. 81801954).

\section{Availability of data and materials}

The datasets used and/or analyzed during the current study are available from the corresponding author on reasonable request.

\section{Authors' contributions}

SX and JH conceived the study and designed the experiments. WY and HS performed the experiments and drafted the manuscript. JX, WJ and GK made substantial contributions to data analysis. All authors read and approved the final manuscript.

\section{Ethics approval and consent to participate}

All animal procedures were approved by The Ethics Committee of Clinical Research, Renmin Hospital of Wuhan University (reference no. WDRY2018-K048). All animal experiments were performed in compliance with the Guidelines for Proper Conduct of Animal Experiments established by the Science Council.

\section{Patient consent for publication}

Not applicable.

\section{Competing interests}

The authors declare that they have no competing interests.

\section{References}

1. ARDS Definition Task Force; Ranieri VM, Rubenfeld GD Thompson BT, Ferguson ND, Caldwell E, Fan E, Camporota L and Slutsky AS: Acute respiratory distress syndrome: The Berlin Definition. JAMA 307: 2526-2533, 2012.

2. Matthay MA, Ware LB and Zimmerman GA: The acute respiratory distress syndrome. J Clin Invest 122: 2731-2740, 2012.

3. Butt Y, Kurdowska A and Allen TC: Acute lung injury: A clinical and molecular review. Arch Pathol Lab Med 140 345-350, 2016.

4. Wong JJ, Jit M, Sultana R, Mok YH, Yeo JG, Koh JWJC, Loh TF and Lee JH: Mortality in pediatric acute respiratory distress syndrome: A systematic review and meta-analysis. J Intensive Care Med 34: 563-571, 2019.

5. Keim G, Watson RS, Thomas NJ and Yehya N: New morbidity and discharge disposition of pediatric acute respiratory distress syndrome survivors. Crit Care Med 46: 1731-1738, 2018.

6. Graves PR, Siddiqui F, Anscher MS and Movsas B: Radiation pulmonary toxicity: From mechanisms to management. Semin Radiat Oncol 20: 201-207, 2010

7. Miravitlles M, Cosio BG, Arnedillo A, Calle M, Alcazar-Navarrete B, Gonzalez C, Esteban C, Trigueros JA, Rodriguez Gonzalez-Moro JM, Quintano Jimenez JA and Baloira A: A proposal for the withdrawal of inhaled corticosteroids in the clinical practice of chronic obstructive pulmonary disease. Respir Res 18: 198, 2017.
8. Wilson JG, Liu KD, Zhuo H, Caballero L, McMillan M, Fang X, Cosgrove K, Vojnik R, Calfee CS, Lee JW, et al: Mesenchymal stem (stromal) cells for treatment of ARDS: A phase 1 clinical trial. Lancet Respir Med 3: 24-32, 2015.

9. Zhu Y, Liu T, Song K, Fan X, Ma X and Cui Z: Adipose-derived stem cell: A better stem cell than BMSC. Cell Biochem Funct 26: 664-675, 2008.

10. Zheng G, Huang L, Tong H, Shu Q, Hu Y, Ge M, Deng K, Zhang L, Zou B, Cheng $\mathrm{B}$ and $\mathrm{Xu}$ J: Treatment of acute respiratory distress syndrome with allogeneic adipose-derived mesenchymal stem cells: A randomized, placebo-controlled pilot study. Respir Res 15: 39, 2014

11. Pittenger MF, Mackay AM, Beck SC, Jaiswal RK, Douglas R, Mosca JD, Moorman MA, Simonetti DW, Craig S and Marshak DR: Multilineage potential of adult human mesenchymal stem cells. Science 284: 143-147, 1999.

12. Vellasamy S, Sandrasaigaran P, Vidyadaran S, George E and Ramasamy R: Isolation and characterisation of mesenchymal stem cells derived from human placenta tissue. World J Stem Cells 4: 53-61, 2012.

13. Zhao Y, Gillen JR, Harris DA, Kron IL, Murphy MP and Lau CL: Treatment with placenta-derived mesenchymal stem cells mitigates development of bronchiolitis obliterans in a murine model. J Thorac Cardiovasc Surg 147: 1668-1677.e1665, 2014.

14. Huang X, Xiu H, Zhang S and Zhang G: The role of macrophages in the pathogenesis of ALI/ARDS. Mediators Inflamm 2018: 1264913,2018

15. Wynn TA, Chawla A and Pollard JW: Macrophage biology in development, homeostasis and disease. Nature 496: 445-455, 2013.

16. Abumaree MH, Al Jumah MA, Kalionis B, Jawdat D, Al Khaldi A, Abomaray FM, Fatani AS, Chamley LW and Knawy BA: Human placental mesenchymal stem cells (pMSCs) play a role as immune suppressive cells by shifting macrophage differentiation from inflammatory M1 to anti-inflammatory M2 macrophages. Stem Cell Rev Rep 9: 620-641, 2013.

17. Lee HH, Jeong JW, Hong SH, Park C, Kim BW and Choi YH: Diallyl trisulfide suppresses the production of lipopolysaccharide-induced inflammatory mediators in BV2 microglia by decreasing the NF-kappaB pathway activity associated with toll-like receptor 4 and CXCL12/CXCR4 pathway blockade. J Cancer Prev 23: 134-140, 2018.

18. Janssens R, Struyf S and Proost P: Pathological roles of the homeostatic chemokine CXCL12. Cytokine Growth Factor Rev 44: 51-68, 2018.

19. Buttenschoen K, Kornmann M, Berger D, Leder G, Beger HG and Vasilescu C: Endotoxemia and endotoxin tolerance in patients with ARDS. Langenbecks Arch Surg 393: 473-478, 2008.

20. Chen $\mathrm{H}$, Bai $\mathrm{C}$ and Wang $\mathrm{X}$ : The value of the lipopolysaccharide-induced acute lung injury model in respiratory medicine. Expert Rev Respir Med 4: 773-783, 2010.

21. Livak KJ and Schmittgen TD: Analysis of relative gene expression data using real-time quantitative PCR and the 2(-Delta Delta C(T)) method. Methods 25: 402-408, 2001.

22. Li G, Zhou CL, Zhou QS and Zou HD: Galantamine protects against lipopolysaccharide-induced acute lung injury in rats. Braz J Med Biol Res 49: e5008, 2016.

23. Zhao YF, Luo YM, Xiong W, Ding W, Li YR, Zhao W, Zeng HZ, Gao HC and Wu XL: Mesenchymal stem cell-based FGF2 gene therapy for acute lung injury induced by lipopolysaccharide in mice. Eur Rev Med Pharmacol Sci 19: 857-865, 2015.

24. Li JW and Wu X: Mesenchymal stem cells ameliorate LPS-induced acute lung injury through KGF promoting alveolar fluid clearance of alveolar type II cells. Eur Rev Med Pharmacol Sci 19: 2368-2378, 2015.

25. D'Alessio FR, Tsushima K, Aggarwal NR, West EE, Willett MH, Britos MF, Pipeling MR, Brower RG, Tuder RM, McDyer JF and King LS: CD4+CD25+Foxp3+ Tregs resolve experimental lung injury in mice and are present in humans with acute lung injury. J Clin Invest 119: 2898-2913, 2009.

26. Xie S, Tu Z, Xiong J, Kang G, Zhao L, Hu W, Tan H, Tembo KM, Ding Q, Deng X, et al: CXCR4 promotes cisplatin-resistance of non-small cell lung cancer in a CYP1B1-dependent manner. Oncol Rep 37: 921-928, 2017.

27. Tu Z, Xie S, Xiong M, Liu Y, Yang X, Tembo KM, Huang J, Hu W Huang X, Pan S, et al: CXCR4 is involved in CD133-induced EMT in non-small cell lung cancer. Int J Oncol 50: 505-514, 2017.

28. Xie S, Zeng W, Fan G, Huang J, Kang G, Geng Q, Cheng B, Wang $\mathrm{W}$ and Dong P: Effect of CXCL12/CXCR4 on increasing the metastatic potential of non-small cell lung cancer in vitro is inhibited through the downregulation of CXCR4 chemokine receptor expression. Oncol Lett 7: 941-947, 2014. 
29. Johnston LK, Rims CR, Gill SE, McGuire JK and Manicone AM: Pulmonary macrophage subpopulations in the induction and resolution of acute lung injury. Am J Respir Cell Mol Biol 47: 417-426, 2012

30. Zheng G, Ge M, Qiu G, Shu Q and Xu J: Mesenchymal stromal cells affect disease outcomes via macrophage polarization. Stem Cells Int 2015: 989473, 2015.

31. MokhberDezfouliMR,JabbariFakhrM,Sadeghian ChaleshtoriS, Dehghan MM, Vajhi A and Mokhtari R: Intrapulmonary autologous transplant of bone marrow-derived mesenchymal stromal cells improves lipopolysaccharide-induced acute respiratory distress syndrome in rabbit. Crit Care 22: 353, 2018.

32. Horwitz EM, Gordon PL, Koo WK, Marx JC, Neel MD, McNall RY, Muul L and Hofmann T: Isolated allogeneic bone marrow-derived mesenchymal cells engraft and stimulate growth in children with osteogenesis imperfecta: Implications for cell therapy of bone. Proc Natl Acad Sci USA 99: 8932-8937, 2002.

33. Jerkic M, Masterson C, Ormesher L, Gagnon S, Goyal S, Rabani R, Otulakowski G, Zhang H, Kavanagh BP and Laffey JG: Overexpression of IL-10 enhances the efficacy of human umbilical-cord-derived mesenchymal stromal cells in E. coli Pneumosepsis. J Clin Med 8: E847, 2019.

34. Zhang X and Dong S: Protective effects of erythropoietin towards acute lung injuries in rats with sepsis and its related mechanisms. Ann Clin Lab Sci 49: 257-264, 2019.

35. Badamjav R, Sonom D, Wu Y, Zhang Y, Kou J, Yu B and Li F: The protective effects of Thalictrum minus L. on lipopolysaccharide-induced acute lung injury. J Ethnopharmacol 248 : $112355,2020$.

36. Liu J, Chang G, Huang J, Wang Y, Ma N, Roy AC and Shen X: Sodium butyrate inhibits the inflammation of lipopolysaccharide-induced acute lung injury in mice by regulating the toll-like receptor 4/nuclear factor $\kappa \mathrm{B}$ signaling pathway. J Agric Food Chem 67: 1674-1682, 2019.

37. Lu Y, Xu D, Liu J and Gu L: Protective effect of sophocarpine on lipopolysaccharide-induced acute lung injury in mice. Int Immunopharmacol 70: 180-186, 2019.
38. Aggarwal NR, King LS and D'Alessio FR: Diverse macrophage populations mediate acute lung inflammation and resolution. Am J Physiol Lung Cell Mol Physiol 306: L709-L725, 2014.

39. Lomas-Neira J, Chung CS, Perl M, Gregory S, BiW and Ayala A Role of alveolar macrophage and migrating neutrophils in hemorrhage-induced priming for ALI subsequent to septic challenge. Am J Physiol Lung Cell Mol Physiol 290: L51-L58, 2006.

40. Yang H, Lu Z, Huo C, Chen Y, Cao H, Xie P, Zhou H, Liu D, Liu J and Yu L: Liang-Ge-San, a classic traditional Chinese medicine formula, attenuates lipopolysaccharide-induced acute lung injury through up-regulating miR-21. Front Pharmacol 10: $1332,2019$.

41. Tang J, Diao P, Shu X, Li L and Xiong L: Quercetin and quercitrin attenuates the inflammatory response and oxidative stress in LPS-induced RAW264.7 cells: In vitro assessment and a theoretical model. Biomed Res Int 2019: 7039802, 2019.

42. Luo XY, Meng XJ, Cao DC, Wang W, Zhou K, Li L, Guo M and Wang P: Transplantation of bone marrow mesenchymal stromal cells attenuates liver fibrosis in mice by regulating macrophage subtypes. Stem Cell Res Ther 10: 16, 2019.

43. Feng Y, Xu Q, Yang Y, Shi W, Meng W, Zhang H, He X, Sun M, Chen Y, Zhao J, et al: The therapeutic effects of bone marrow-derived mesenchymal stromal cells in the acute lung injury induced by sulfur mustard. Stem Cell Res Ther 10: 90, 2019.

44. Liu F, Qiu H, Xue M, Zhang S, Zhang X, Xu J, Chen J, Yang Y and Xie J: MSC-secreted TGF- $\beta$ regulates lipopolysaccharide-stimulated macrophage M2-like polarization via the Akt/FoxO1 pathway. Stem Cell Res Ther 10: 345, 2019.

This work is licensed under a Creative Commons Attribution-NonCommercial-NoDerivatives 4.0 International (CC BY-NC-ND 4.0) License. 